\title{
SCREENING OF TICK-BORNE ENCEPHALITIS VIRUS METHYLTRANSFERASE INHIBITORS IN VITRO AND IN SILICO
}

\section{V.S. Frolenko, ${ }^{1,2}$, V.I. Uvarova', A.A. Nikitina', E.V. Khvatov', M. Dodina', E. Bayurova', L.I. Kozlovskaya ${ }^{1,2}$, D.I. Osolodkin ${ }^{1,2,3}$}

\author{
${ }^{1} \mathrm{FSASI}$ «Chumakov FSC R\&D IBP RAS» (Institute of Polyomyelitis), \\ 108819, Moscow, Settlement 'Moscovskiy', \\ Village of Institute of Poliomyelitis, Premises 8, bldg. 1. \\ ${ }^{2}$ Sechenov First MSMU, 119992, Moscow, Trubetskaya street, 8, bldg. 2. \\ ${ }^{3}$ Department of Chemistry, Lomonosov MSU, \\ 119991, Russia, Moscow, Leninskie Gory, 1, bldg. 3.
}

DOI: 10.19163/MedChemRussia2021-2021-323

E-mail:vasilisa.frolenko@gmail.com

Tick-borne encephalitis (TBE) is a viral disease transmitted by ticks and caused by tick-borne encephalitis virus (TBEV). TBEV is a member of the genus Flavivirus in the family of Flaviviridae and is the most important of flaviviruses transmitted by ticks. Due to relatively little prevalence, TBEV is less common and researched virus than mosquito-borne flaviviruses such as dengue virus, West Nile virus and yellow fever virus. Nowadays there is a necessity for developing specific drugs against TBE due to severity of the disease and difficulties in vaccine application. It was suggested that viral proteins, especially enzymes, are potential antiviral drug targets. Methyltransferase (MT) is the enzyme that catalyzes viral RNA capping and is considered to be as one of the main target. Cap structure provides resistance to cellular enzymes and contributes to mRNA translation.

The main aim of current work was to find potential TBEV MT inhibitors. Activity prediction was carried out with using a Random Forest model implemented in scikit-learn library. To build this model we have used the results of high throughput screening of inhibitors (available in PubChem BioAssay database) against MT enzyme of dengue virus 2. The data included 338,000 compounds, where 1013 were active. Model evaluation was performed by the standard classification metrics such as Precision, Recall and F1-score. To evaluate model applicability on imbalanced datasets we comparatively analyzed $R O C$ and Precision-Recall curves. The model showed acceptable prediction quality.

We have used our model to predict the activity of compounds from the Chumakov Center collection (19 compounds out of 1125 were classified as active) against TBEV MT. According to the previous experiments, 9 compounds from the hit list showed antiviral activity in vitro. We have established an experimental method to detect inhibitory activity of certain compounds against TBEV using such technique as Thermal Shift Assay (TSA). Positive hits that were identified by this assay can be considered as potential lead compounds and will be used in future for developing new drugs against TBE. 